\title{
Benefícios da cirurgia cardíaca na qualidade de vida de pacientes adultos e idosos
}

Benefits of cardiac surgery in the quality of life of adult and elderly patients

FisiSenectus . Unochapecó Ano 6, n. 2 - Jul/Dez. 2018 p. $4-15$

Débora D'Agostini Jorge Lisboa. debydj@yahoo.com.br.

Fisioterapeuta. Mestre em Envelhecimento Humano pela Universidade de Passo Fundo (UPF-RS). Especialista em Fisioterapia em Terapia intensiva. Fisioterapeuta e coordenadora do setor de fisioterapia do Hospital de Clínicas de Passo Fundo/RS. Docente do curso de fisioterapia da UPF-RS

Eliane Lúcia Colussi. colussi@upf.br.

Historiadora. Doutora em História pela PUC-RS. Docente do curso de História e do Programa de Mestrado em Envelhecimento Humano da Universidade de Passo Fundo (UPF-RS).

Marlene Doring. doring@upf.br.

Enfermeira. Doutora em saúde pública. Docente do Programa de Mestrado em Envelhecimento Humano da Universidade de Passo Fundo (UPF-RS).

Renato Ravizon Lisboa. rrlisboa@yahoo.com.br.

Médico. Especialista em Medicina de Família e Comunidade pela Sociedade Brasileira de Medicina de Família e Comunidade (SBMFC/AMB). Especialista em Clínica Médica pela SBCM/AMB. Médico clínico da Prefeitura Municipal de Passo Fundo/RS e da Prefeitura Municipal de Marau/RS.

Gabriela Colussi. gabriela_colussi@icloud.com.

Psicóloga autônoma.

Luana Battistella. lu_battistella@hotmail.com.

Psicóloga autônoma.

Indaiara Medeiros da Silva. daiamedeirospf@hotmail.com.br.

Fisioterapeuta no Hospital da Unimed/SC.

Júlia Mognon. juh_mognon@hotmail.com.

Fisioterapeuta da prefeitura de Bento Gonçalves/RS.

Isadora Rebolho Sisto. isadora_sisto@hotmail.com.

Fisioterapeuta. Mestre em ciências da reabilitação pela Fundação Universidade Federal de Ciências da Saúde de Porto Alegre/RS (UFCSPA). Fisioterapeuta do Instituto de Cardiologista Porto Alegre/RS.

\section{Resumo}

Introdução: A cirurgia cardíaca é um tratamento efetivo na doença arterial coronariana. Esse tratamento proporciona melhora na qualidade de vida dos pacientes. O questionário de qualidade de vida SF-36 permite monitorar as condições de saúde antes e após o tratamento cirúrgico, sensível à melhora clínica. Objetivos: Avaliar o impacto da cirurgia cardíaca na qualidade de vida dos pacientes adultos e idosos. Métodos: Estudo 
de coorte prospectivo não controlado, realizado em dois hospitais de grande porte. A coleta ocorreu através do questionário SF-36, em três momentos distintos, pré, pós e três meses após o procedimento. Realizouse análise descritiva e inferencial dos dados. 0 nível de significância foi de 0,05. Resultados: A amostra foi composta por 70 indivíduos. Na capacidade funcional, o escore da mediana pré foi de 40,0, pós 15,0 e seguimento 70,0. No domínio limitação por aspectos físicos, o escore da mediana no pré e pós foi zero e três meses foi 25,0. Em relação à dor, o escore da mediana pré 52,0, pós 32,0 e seguimento 62,0. Na vitalidade, 0 escore da mediana pré e pós foi 50,0 e seguimento três meses 70,0. No aspecto social, o escore da mediana pré foi 62,5 , pós 50,0 e seguimento 87,5. A limitação por aspectos emocionais, o escore da mediana pré 33,3, pós zero e após três meses 100,0. Observou-se significância p<0,05 em todos os domínios avaliados. Conclusão: A cirurgia cardíaca tem um impacto positivo na qualidade de vida dos pacientes em todos os domínios avaliados, especialmente após três meses de procedimento.

\title{
Palavras-chave
}

Qualidade de vida; Procedimentos Cirúrgicos Cardiovasculares; Hospitalização.

\begin{abstract}
Introduction: Cardiac surgery is an effective means for the treatment of coronary artery disease. In addition, this treatment provides improved quality of life for patients. The SF-36 quality of life questionnaire enables monitoring health conditions before and after surgery, being sensitive to clinical improvement. Objective: Evaluate the impact of cardiac surgery quality of life of adults and elderly patients. Methods: It is an uncontrolled prospective cohort study in two large hospitals in Passo Fundo/RS. Data collection occurred through the SF-36 questionnaire, in three different times, preoperative, postoperative during hospitalization and after three months of surgery. A descriptive and inferential analysis. The significance was observed $p<0.05$. Results: Functional capacity, the score pre-median was 40.0, post 15.0 and follow-up 70.0. In the limitation domain by physical aspects, the median score in the pre and post was zero and three months was 25.0. With regard to pain, the median score pre 52.0, post 32.0 and the follow-up 62.0. In vitality, the median score in the pre and post was 50.0 and in the follow-up 70.0. In the social aspect, the pre-median score was 62.5, post 50.0 and follow-up 87.5. The limitation by emotional aspects, the score pre-median was 33.3, in the postoperative zero and after three months 100.0. Conclusion: It is concluded that cardiac surgery has a positive impact on the quality of life of patients in all domains evaluated, especially after three months of procedure.
\end{abstract}

\section{Keywords}

Quality of Life; Cardiovascular Surgical Procedures; Hospitalization.

\section{Introdução}

$\infty \times \infty \times \infty \times \infty \times \infty \times \infty \times \infty \times \infty \times \infty \times \infty \times \infty \times \infty \times \infty \times \infty$

Estudos mostraram uma maior incidência de eventos cardiovasculares na população com sintomas depressivos e baixos escores de qualidade de vida, assim, o estilo de vida e o estresse emocional são fatores de risco para doenças cardiovasculares e têm merecido destaque na literatura ${ }^{1}$.

As doenças cardiovasculares estão diretamente relacionadas ao crescente número de pacientes que precisam ser submetidos a procedimentos cirúrgicos cardíacos. Essas intervenções afetam diretamente questões físicas, de produtividade, sociais e emocionais, além de trazerem sequelas que podem acarretar difíceis mudanças de comportamento e hábitos de vida, comprometendo a qualidade de vida dos pacientes ${ }^{2}$.

A alta prevalência de doença cardíaca na população tem grandes implicações no período pós-operatório e o risco de eventos cardíacos graves pode ser significante para pacientes submetidos a grandes cirurgias ${ }^{3}$. Se, por um lado, o crescente desenvolvimento tecnológico da medicina possibilitou o tratamento de várias doenças, garantindo maior longevidade à população, por outro lado, desencadeou um processo de fragilização, no qual 
se percebe aumento da longevidade muitas vezes com baixa qualidade de vida. Portanto, a preocupação com o conceito de qualidade de vida vem para resgatar aspectos psicológicos, físicos e sociais, além dos econômicos ${ }^{4}$.

A Qualidade de Vida(QV) é, sem dúvida, um dos temas mais comentados e discutidos na atualidade ${ }^{4}$. A definição global de qualidade de vida considera as condições externas de vida e as experiências subjetivas do indivíduo. Um estudo sobre a qualidade de vida em pacientes com doença aterosclerótica coronariana grave e estável indica que não é uma tarefa fácil quantificar a qualidade de vida, para tanto, existem várias propostas de instrumentos que foram validados por diversos estudos e traduzidos para o Brasil. Entre eles, podem ser citados o Medical Outcomes Study 36-item Short-form Health Survey (SF-36) como um instrumento mais abrangente e bem aplicado a pacientes com doenças crônicas ${ }^{1}$.

Diante desse contexto, este estudo tem como objetivo avaliar o impacto da cirurgia cardíaca na qualidade de vida dos pacientes adultos e idosos.

\section{Método}

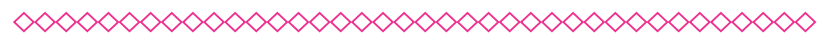

Estudo de coorte prospectivo não controlado com adultos e idosos submetidos à cirurgia cardíaca, acompanhados no período de três meses após o procedimento cirúrgico, desenvolvido nas dependências de dois hospitais de grande porte, do município de Passo Fundo/RS, junto à unidade de internação, primeira fase da coleta, correspondente à avaliação pré-operatória, no Centro de Tratamento Intensivo (CTI) Cardiológico e/ou Unidade de Terapia Intensiva (UTI), correspondente à segunda fase da avaliação pós-operatória, e no ambulatório da Cardiologia ou por contato telefônico, conforme preferência dos participantes, terceira fase da coleta, correspondente a três meses após a cirurgia cardíaca.

Dentre as cirurgias cardíacas realizadas neste período, foram incluídos na amostra 86 pacientes, consecutivamente, entre o período de primeiro de maio a 31 de julho de 2015. Foram incluídos na amostra todos os indivíduos adultos e idosos, de ambos os sexos, submetidos à cirurgia cardíaca que ficaram internados nos dois hospitais de Passo Fundo. Foram critérios de exclusão pacientes com deficiência ou comprometimento auditivo, fonativo e/ou mental grave que interferiram na realização dos questionários; pacientes que se submeteram à reintervenção cirúrgica não cardíaca; e pacientes submetidos à cirurgia cardíaca de urgência.

O desenvolvimento do estudo ocorreu em conformidade com o preconizado pela Resolução 466/12 do Conselho Nacional de Saúde (CNS) de 12 de dezembro de 2012 . o projeto foi submetido e aprovado pelo Comitê de Ética em pesquisa da Universidade de Passo Fundo (UPF), com o protocolo número 1.029.446, e aprovado pelas comissões de Pesquisa dos dois hospitais. Os participantes que aceitaram participar do estudo assinaram o Termo de Consentimento Informado Livre e Esclarecido (TCLE).

A coleta de dados foi realizada pela pesquisadora e cinco entrevistadoras (duas bolsistas de Iniciação Científica do Curso de Psicologia; duas fisioterapeutas residentes do Programa de Residência Multiprofissional (PRM) em cardiologia e uma fisioterapeuta voluntária) por meio de entrevista individual e consulta ao prontuário dos participantes. Antes do início dos procedimentos, todas as entrevistadoras foram treinadas e qualificadas para a aplicação dos questionários. Foram realizadas sessões de treinamento para esclarecimento de dúvidas e forma correta da utilização e preenchimentos do questionário sociodemográfico e da escala SF-36.

Para avaliação da qualidade de vida foi utilizada a versão adaptada para o português do instrumento genérico Medical Outcomes Study 36 - item Short Form (SF-36), idealizado por Ware e Sherboune e validado para o português por Ciconelli ${ }^{5}$. Este questionário é uma ferramenta que pode ser aplicada em pessoas a partir de 12 anos de idade e que objetiva pesquisar o estado de saúde física e mental na prática clínica individualizada e na população geral. É um questionário multidimensional composto por 36 itens que abordam oito domínios em dois grandes componentes: o componente físico que envolve a capacidade funcional (com dez itens), dor (dois itens), estado geral de saúde (cinco itens), aspectos físicos (quatro itens). 
O componente mental contempla a saúde mental (cinco itens), os aspectos emocionais (três itens), a vitalidade (quatro itens) e os aspectos sociais (dois itens), sendo avaliado por 35 questões. Além disso, há mais uma questão para a avaliação comparativa do estado geral de saúde atual e de um ano atrás. A finalidade das questões foi transformar medidas subjetivas em dados objetivos, que permitissem análises de forma específica, global e reprodutível. 0 instrumento aborda as últimas quatro semanas.

Para a avaliação dos resultados, utilizou-se 0 escore próprio da cada questão, o qual avalia tanto os aspectos negativos (doença/enfermidade) como os pontos positivos (bem-estar). Um escore numérico baixo reflete a má percepção da saúde, a perda da função e a presença de dor, enquanto o escore numérico alto reflete a boa percepção da saúde, função preservada e ausência de dor do sujeito. Para a avaliação dos resultados, as respostas aos seus itens são computadas em seus respectivos componentes, e esses valores são normatizados em uma escala de zero a 100. Menores valores refletem uma percepção de saúde precária e dor (pior avaliação), enquanto altos valores refletem uma percepção de boa saúde, ausência de déficits funcionais e de dor (melhora a avaliação da qualidade de vida).

Na análise dos dados, as variáveis categóricas foram descritas como frequência absoluta e relativa. As variáveis numéricas foram descritas como média \pm desvio padrão ou mediana (percentil ${ }_{25}$ - percentil $_{75}$ ), conforme apresentarem distribuição normal ou não normal. E, para avaliar a variação da qualidade de vida ao longo do tempo, utilizou-se análise de variância, na qual o efeito de tempo (basal, pós-cirurgia e no seguimento de três meses) de cada um dos domínios do SF-36 foi especificado como efeito intra-sujeito. Para comparações múltiplas, aos pares, utilizou-se análise de contrastes simples. Consideraram-se como estatisticamente significativos testes com valor de probabilidade $<0,05$.

\section{Resultados}

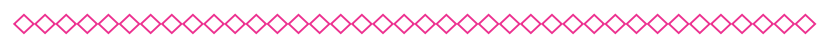

Dos 93 pacientes que realizaram cirurgia cardíaca no período de primeiro de maio a 31 de julho de 2015 nas instituições em estudo, sete pacientes foram excluídos da amostra por se enquadrarem nos critérios de exclusão, seis por cirurgia de urgência e um por reintervenção. Assim, 86 pacientes correspondiam à cirurgia eletiva e foram incluídos na amostra. Destes, foram excluídos oito pacientes $(9,3 \%)$ por óbito, dois $(2,3 \%)$ por AVC intra-hospitalar e seis $(7,0 \%)$ por perda de seguimento, assim, $70(81,4 \%)$ completaram o seguimento de três meses.

A Tabela 1 descreve as características sociodemográficas da população em estudo. A idade média da população em estudo é de 59,9 anos $( \pm 14,0)$. Dos participantes do estudo $44(62,9 \%)$ são do sexo masculino, $47(67,1 \%)$ são casados e $48(68,6 \%)$ são aposentados. A religião mais encontrada foi a católica com 57 (81,4\%). A raça mais prevalente foi a branca, com $62(88,6 \%)$ participantes.

A Tabela 2 descreve as características clínicas da população em estudo. Dos pacientes submetidos à cirurgia cardíaca, constatou-se que as doenças de base mais prevalentes são Diabetes, Hipertensão, Doença Arterial Coronariana (DAC) e um menor número de pacientes tem Doença Pulmonar Obstrutiva Crônica (DPOC) e Insuficiência Renal Crônica (IRC). Dos pacientes avaliados, $11,4 \%$ são tabagistas e 25,7\% ex-tabagistas.

A Tabela 3 descreve os domínios do SF-36 pré-operatório, pós-cirurgia e no seguimento de três meses. Observou-se que, na capacidade funcional pós-operatório, houve uma queda do escore em relação ao pré-operatório, enquanto no seguimento de três meses, registou-se um aumento no escore da capacidade funcional. No domínio limitação por aspectos físicos, o escore inicial foi de zero, mantendo-se o mesmo valor encontrado na fase pós-operatória, já no seguimento de três meses, o vaIor aumentou a pontuação do escorre. Em relação à dor, obteve-se piora da dor do pós-operatório em relação ao pré-operatório, com aumento do escore da dor no seguimento de três meses. 0 estado geral de saúde se agravou no pós-operatório em relação ao pré-operatório, com melhora do escore no seguimento de três meses. Na vitalidade, os valores encontrados no pré-operatório mantiveram-se no pós-operatório, com melhora no seguimento de três meses. No aspecto social, observou-se piora dos resultados no pós-operatório, com melhora destes valores no seguimento de três meses. Com relação à limitação por aspectos emocionais, no pós-operatório ocorreu uma piora dos resultados 
em relação ao pré-operatório, alcançando, no seguimento de três meses um aumento destes valores. Na saúde mental, observou-se melhora dos resultados no seguimento de três meses em relação ao pré e ao pós-operatório.

Observou-se variação significativa do domínio capacidade funcional ao longo do tempo $(p<0,001)$. Apesar de uma redução significativa do escore pós- cirurgia em relação ao basal, com diferença média de 25,4 (IC95\% 18,9 - 32,0, p<0,001), houve uma recuperação no seguimento de três meses, havendo diferença média em relação ao pós-cirurgia de 40,7 (IC 95\% 32,7-48,7, p<0,001), atingindo valores mais elevados que o basal, com diferença média de 15,3 (IC95\% 7,7-22,9, p<0,001).

No domínio limitação por aspectos físicos, observou-se uma variação significativa ao longo do tempo $(p<0,001)$. Ocorreu uma redução não estatisticamente significativa do escore no pós-cirurgia em relação ao basal, e diferença média de 7,1 (IC95\% -0,9-15,1, p=0,079), um aumento estatisticamente significativo no seguimento de três meses em relação ao pós-cirurgia, diferença média de 23,6 (IC95\% 12,8 - 34,4, p<0,001), valor também significativamente mais elevado que 0 escore basal, com diferença média de 16,4 (IC95\% $3,1-29,8, p=0,017)$.

Observou-se variação significativa do domínio dor ao longo do tempo $(p<0,001)$. Apesar de uma redução significativa do escore pós-cirurgia em relação ao basal, diferença média 13,7 (IC95\% 6,0 - 21,3, p=0,001), houve uma recuperação no seguimento de 3 meses, diferença média em relação ao pós-cirurgia 28,5 (IC 95\% 22,4 - 34,5, $p<0,001$ ), atingindo valores mais elevados que o basal, diferença média 14,8 (IC95\% 6,2 - 23,4, p=0,001).

Notou-se uma variação significativa do domínio estado geral de saúde ao longo do tempo $(p=0,004)$. Houve um aumento não estatisticamente significativo do escore no pós-cirurgia em relação ao basal, com diferença média de 1,0 (IC95\% - 5,0 - 6,0, $p=0,841$ ), um aumento estatisticamente significativo no seguimento de três meses em relação ao pós-cirurgia, com diferença média de 7,4 (IC95\% 2,2 - 12,6, p =0,006), também significativamente mais elevado que o escore basal, com diferença média de 8,0 (IC95\% 2,9-13,0, $p=0,002$ ).

Observou-se variação significativa do domínio vitalidade ao longo do tempo $(p<0,001)$. Houve uma redução não estatisticamente significativa do escore no pós-cirurgia em relação ao basal, com diferença média de 0,6 (IC95\% -5,4-6,7, p=0,833), um aumento estatisticamente significativo no seguimento de três meses em relação ao pós-cirurgia, com diferença média de 16,4 (IC95\% 10,5 - 22,2, $p<0,001$ ), também significativamente mais elevado que o escore basal, com diferença média de 15,7 (IC95\% 9,9-21,5, p<0,001).

Variação significativa do domínio aspectos sociais do tempo $(p<0,001)$ foi percebida. Houve uma redução não estatisticamente significativa do escore no pós-cirurgia em relação ao basal, com diferença média de 2,5 (IC95\% -5,4-10,4, $p=0,531$ ), um aumento estatisticamente significativo no seguimento de três meses em relação ao pós-cirurgia, com diferença média de 21,1 (IC95\% 13,6 - 28,6, $p<0,001$ ), também significativamente mais elevado que o escore basal, com diferença média de 18,6 (IC95\% 10,6-26,5, p<0,001).

No domínio limitação por aspectos emocionais, observou-se variação significativa ao longo do tempo $(p<0,001)$. Apesar de uma redução significativa do escore pós-cirurgia em relação ao basal, com diferença média de 11,4 (IC95\% 1,1 - 21,8, p=0,031), houve uma recuperação no seguimento de três meses, com diferença média em relação ao pós-cirurgia de 39,1 (IC 95\% 27,3-50,8, p<0,001), atingindo valores mais elevados que o basal, com diferença média de 27,6 (IC95\% 15,0 - 40,2, p<0,001).

Observou-se variação significativa do domínio saúde mental ao longo do tempo $(p<0,001)$. Houve um aumento não estatisticamente significativo do escore no pós-cirurgia em relação ao basal, com diferença média de 3,3 (IC95\% -2,7-9,2, p=0,282), um aumento estatisticamente significativo no seguimento de três meses em relação ao pós-cirurgia, com diferença média de 9,2 (IC95\% 4,5 - 13,9, p<0,001), também significativamente mais elevado que o escore basal, com diferença média de 12,5 (IC95\% 7,0 - 17,9, p=0,001).

\section{Discussão}

$\infty<\infty<\infty<\infty<\infty<\infty<\infty<\infty<\infty<\infty<\infty<\infty<\infty<\infty<\infty<$

Este estudo avaliou a qualidade de vida do paciente submetido à cirurgia cardíaca em três 


\section{Fisi گ̌enenectus}

diferentes momentos: pré-operatório, pós-operatório e no seguimento de três meses, através do instrumento SF-36. Este instrumento de avaliação analisa dados de saúde física (capacidade funcional, aspectos físicos, dor e estado geral de saúde) e mental (vitalidade, aspecto social, aspecto emocional e saúde mental) e é utilizado para mensurar possíveis modificações na qualidade de vida de determinadas intervenções médicas.

0 presente estudo observou melhora de todos os domínios dos componentes físico ou mental após três meses de cirurgia cardíaca, corroborando com o estudo de Takiuti et al. ${ }^{6}$ onde foi avaliada a qualidade de vida após angioplastia, tratamento clínico ou revascularização cirúrgica do miocárdio e constatou-se melhora de todos os domínios, tanto no componente físico como no componente mental ao final do estudo, essa melhora, foi mais acentuada nos pacientes submetidos à cirurgia cardíaca.

Em outro estudo, realizado por Favarato et al. ${ }^{7}$, com objetivo de avaliar a qualidade de vida em portadores de Doença Arterial Coronariana (DAC) submetidos a um dos três tratamentos: clínico, cirúrgico ou por angioplastia, em três momentos distintos. Estudo de Favarato et al. que buscou comparar a qualidade de vida de pacientes submetidos aos diferentes tratamentos, concluiu que os pacientes submetidos a cirurgia de revascularização do miocárdio apresentaram os piores resultados na avaliação inicial, porém, aos seis e doze meses após o procedimento, ocorreu uma melhora progressiva, superando os pacientes submetidos a tratamento clínico e à angioplastia em quase todas as dimensões do SF-36. Por isso, pode-se considerar que, embora os pacientes cirúrgicos fossem os mais comprometidos em sua qualidade de vida do ponto de vista dos componentes físicos e mentais, a cirurgia proporcionou incremento significativo na qualidade de vida indo ao encontro do presente estudo, no qual se observou que a qualidade de vida piorou no período pós-operatório e uma meIhora significativa da qualidade de vida após três meses do procedimento ${ }^{7}$.

Bahramnezhad et al. ${ }^{8}$ realizou estudo que avaliou a qualidade de vida em pacientes submetidos à angioplastia coronária percutânea, com avaliações realizadas antes, três, seis e doze meses após a cirurgia. Seus resultados mostraram que não houve diferença significativa na qualidade de vida antes do procedimento e três meses após porém, houve melhora na qualidade de vida aos seis meses e doze meses pós-procedimento. Esta ausência na melhora da qualidade de vida três meses após a angioplastia, encontrada no estudo mencionado, contraria os resultados do presente estudo, onde a qualidade de vida melhorou conforme aponta a avaliação realizada três meses após a cirurgia cardíaca.

Foi avaliada a qualidade de vida em pacientes submetidos à cirurgia cardíaca de revascularização com e sem circulação extracorpórea (CEC). Foram observadas importantes modificações tanto no componente físico quanto no mental. No componente físico, os pacientes alcançaram melhora significativa em todos os domínios. Essa mesma condição foi observada no componente mental. Todavia, ao comparar os resultados entre as duas formas terapêuticas (cirurgia cardíaca de revascularização com e sem (EC), essas diferenças não foram perceptíveis ${ }^{9}$.

Gois, Dantas e Torrati ${ }^{10}$ avaliaram a qualidade de vida relacionada à saúde antes e seis meses após o procedimento de revascularização do miocárdio. No estudo, quatro componentes do SF-36 apresentavam valores menores no pré-operatório, demonstrando maior comprometimento nos componentes: aspectos físicos, aspectos emocionais, capacidade funcional e dor. Já na avaliação realizada seis meses após o procedimento cirúrgico, constatou-se melhora estatisticamente significativa na avaliação dos oito componentes do SF-36. Os componentes aspectos físicos e aspectos emocionais foram os que apresentaram melhor avaliação entre os demais. Tais dados vão de encontro aos resultados encontrados no presente estudo.

No estudo realizado por Abelha et al. ${ }^{3}$, no qual foram avaliadas a qualidade de vida e a mortalidade em pacientes com eventos cardíacos graves no pós-operatório em geral, $31 \%$ dos pacientes declaram que sua saúde em geral estava melhor no dia em que completaram o questionário SF-36 do que doze meses antes, enquanto $31 \%$ consideravam sua saúde pior. Os resultados do questionário SF-36 de todos os pacientes que desenvolveram eventos cardíacos graves foram piores em todos 


\section{Fisi Y̌enenectus}

os domínios, exceto em dor corporal e saúde mental. No entanto, esta foi uma limitação do trabaIho, pois os autores não aplicaram o questionário SF-36 antes da cirurgia, de forma que não foi possível comparar a qualidade de vida dos indivíduos antes e depois da cirurgia.

No presente estudo, observou-se uma diminuição significativa no domínio dor ao longo do tempo $(p<0,001)$, corroborando com Choinière et al. ${ }^{11}$, os quais realizaram um estudo multicêntrico prospectivo de dois anos que avaliou a prevalência e os fatores de risco para dor não anginosa persistente após cirurgia cardíaca. Em seu estudo, mostraram que a prevalência de dor pós-operatória diminuiu significativamente ao longo do tempo: $40,1 \%$ aos três meses, $22,1 \%$ aos seis meses, $16,5 \%$ aos 12 meses e $9,5 \%$ aos 24 meses.

No estudo realizado por Araujo ${ }^{12}$, conclui-se que os pacientes submetidos a CRM tiveram menor pontuação nos domínios físico e ambiental, havendo associação entre a presença de fatores de risco e comorbidades com pior avaliação da QV. As questões que envolvem a QV de pacientes no pós-operatório de cirurgia cardíaca possibilitaram a reflexão sobre a real necessidade do paciente revascularizado em relação à sua condição de saúde, QV e satisfação pessoal, o que pode, diretamente, auxiliar os profissionais de saúde envolvidos no planejamento de reabilitação, fornecendo subsídios para implantação de uma assistência voltada para as reais necessidades desses pacientes, com estratégias de educação em saúde, como forma de promover saúde e prevenir agravos eventuais.

Em outro estudo realizado, onde avaliaram a relação entre o domínio meio ambiente, pacientes com renda de até um salário mínimo apresentaram pior avaliação da QV para esse domínio. A faceta recursos financeiros contribuiu para a obtenção dessa avaliação, já que $84 \%$ dos pacientes apresentaram renda inferior a dois salários mínimos. $O$ fato de a maioria ser idosos e aposentados também contribui para essa avaliação. Rendimentos frequentemente diminuídos são fatores socioeconômicos importantes na vida diária e interferem na QV, principalmente em idosos. A baixa renda também deve ser considerada quando se avalia o status clínico do paciente e uso de maior número de medicações ${ }^{13}$.
Pesquisa realizada por Souza e Yamaguchi ${ }^{14}$ observaram que o domínio aspecto sociais foi o que apresentou o segundo maior escore. Segundo esses autores, o suporte social pode adquirir a função de aliviar o estresse em situações de crise, inibir o desenvolvimento de doenças e representar importante papel na recuperação de enfermidades já instaladas, sendo considerado como fator de proteção, assim, constitui foco importante de intervenção junto a essa população específica.

No domínio ambiental, avaliado através do questionário de Whoqol-bref, as questões relacionadas com recursos financeiros, qualidade de cuidados com a saúde, oportunidades de recreação, lazer e adquirir novas habilidades e informações são relevantes para uma melhor ou pior qualidade de vida. Neste estudo, a comprovação de este domínio associar-se à redução de qualidade de vida ocorreu entre os idosos do grupo AG. Estes apresentaram somatória de fatores contribuintes para este resultado. Quanto à menor prática de atividades físicas colaborando para pior qualidade de vida, constata-se o contrário entre os participantes do grupo SESC. Além disso, estes idosos também realizam outras atividades sociais e de lazer, tais como: cursos de pintura, dança, palestras educativas, viagens culturais, entre outras. Isso sugere que outros fatores, além da atividade física por si só, permitem o envelhecimento com melhor qualidade de vida ${ }^{13}$.

Em relação ao domínio psicológico, os pacientes revelaram ter melhor percepção da QV. Todas as facetas contribuíram para esse resultado, com exceção da faceta de sentimentos negativos. Os sentimentos negativos provocam alterações fisiológicas com impacto negativo no prognóstico de DAC e podem influenciar diretamente na adesão ao tratamento que requer mudanças comportamentais. A QV é uma questão de importância e que preocupa os profissionais de enfermagem requerendo desses uma avaliação fidedigna que permita não somente mensurar o impacto de um procedimento cirúrgico cardíaco sobre a vida do paciente mas também oferecer subsídios com base na percepção geral do próprio indivíduo sobre seu estado de saúde ${ }^{15}$.

Dos pacientes submetidos à Revascularização do Miocárdio (RVM) ou à Angioplastia Coronária 


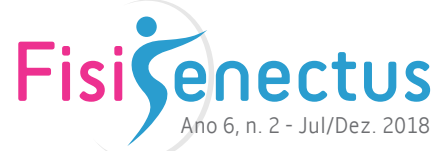

Transluminal Percutânea (ACTP), ao final do sexto mês de seguimento houve melhora da QV. Ainda que a RVM tenha determinado melhora em um maior número de domínios quando comparada à ACTP, esta diferença não foi significativa. $\mathrm{Na}$ avaliação inicial da QV, o grupo submetido à RVM apresentou melhores escores nos domínios vitalidade, social, emocional e mental, enquanto o grupo submetido à ACTP apresentou melhores escores nos domínios dor e saúde. Na comparação da saúde atual em relação ao ano anterior, a ACTP foi superior à RVM ${ }^{16}$.

No estudo de Lima et al. ${ }^{17}$, voltado a avaliar a qualidade de vida de indivíduos idosos cardiopatas pré e pós-cirurgia cardíaca, apontou para um impacto positivo na qualidade de vida antes e após a cirurgia cardíaca nos domínios físico, social e qualidade de vida geral nos participantes em estudo, corroborando com o presente estudo.

Em outro estudo, constatou-se que a avaliação da QV relacionada a saúde (QVRS) pré-operatória deve ser uma parte importante do exame pré-operatório, especialmente nos pacientes de alto risco. Os pacientes idosos com risco cardíaco mais elevado têm menor QV no pré-operatório, mas, mostram uma melhora significante na maioria dos domínios da QVRS após cirurgia cardíaca, em comparação com pacientes mais jovens. 0 grupo de pacientes com idade maior de 70 anos apresentou mais comorbidades pré-operatórias e maior prevalência de complicações pós-operatórias, mas não houve diferença significativa na QVRS comparado com o grupo mais jovem de pacientes um ano após procedimento ${ }^{18}$.

\section{Conclusão}

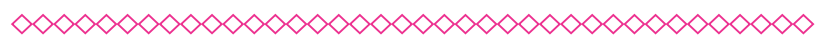

Este estudo mostrou que os pacientes submetidos à cirurgia cardíaca têm uma melhora na qualidade de vida três meses após o procedimento, afinal, em todos os domínios, tanto os componentes da saúde física como os componentes da saúde mental apresentaram resultados significativamente positivos.

\section{Referências}

$\infty<\infty<\infty<\infty<\infty<\infty<\infty<\infty<\infty<\infty<\infty<\infty<\infty<\infty<\infty<\infty$

1. Moriel G, Roscani MG, Matsubara LS, Cerqueira AT de AR, Matsubara BB. Qualidade de Vida em Pacientes com Doença Aterosclerótica Coronariana Grave e Estável. Arq Bras Cardiol. 2010;95(6):691-7.

2. Custódio FM, Gasparino RC. Quality of Life of Patients in the Postoperative Cardiac Surgery Phase. Reme Rev Min Enferm [Internet]. 2013 [cited 2014 Oct 25];17(1):130-4.

3. Abelha FJ, Botelho M, Fernandes V, Barros H. Avaliação da Qualidade de Vida e Mortalidade em Pacientes com Eventos Cardíacos Graves no Pós-Operatório. Rev Bras Anestesiol. 2010;60(3):268-84.

4. Monteiro R, Braile DM, Brandau R, Jatene FB. Qualidade de vida em foco. Rev Bras Cardiovasc. 2010;25(4):568-74.

5. Ciconelli RM, Ferraz MB, Santos W, Meinão I, Quaresma MR. Tradução para a língua portuguesa e validação do questionário genérico de avaliação da qualidade de vida SF-36 (Brasil SF-36). Rev Bras Reum. 1999;39(3):143-50.

6. Takiuti ME, Hueb W, Hiscock SB, Nogueira CRS da R, Girardi P, Fernandes F, et al. Qualidade de vida após Revascularização Cirúrgica do Miocárdio, angioplastia ou Tratamento clínico. Arq Bras Cardiol. 2007;88(5):537-44.

7. Favarato MEC de S, Favarato D, Hueb WA, Aldrighi JM. Qualidade de vida em portadores de doença arterial coronária: comparação entre gêneros. Rev Assoc Med Bras. 2006;52(4):236-41.

8. Bahramnezhad F, Khajeh M, Shiri M, Asgari P, Afshar PF. Quality of Life in Patients Undergoing Percutaneous Transluminal Coronary Angioplasty (PTCA). Glob J Health Sci [Internet]. 2015 Jan [cited 2015 Dec 2];7(5):246-50. Available from: http://www.ncbi.nlm.nih.gov/pubmed/26156913.

9. Nogueira CRSR, Hueb W, Takiuti ME, Priscyla GBMA, Nakano FF, Paulisch F da S., et al. Qualidade de Vida após Revascularização Cirúrgica do 
Miocárdio com e sem Circulação Extracorpórea. Arq Bras Cardiol Bras Cardiol. 2008;91(4):238-44

10. Gois CFL, Dantas RAS, Torrati FG. Qualidade de vida relacionada à saúde antes e seis meses após a revascularização do miocárdio. Rev Gaúcha Enferm. 2009;30(4):700-7.

11. Choinière $M$, Watt-watson J, Victor JC, Baskett RJF, Bussières JS, Carrier M, et al. Prevalence of and risk factors for persistent postoperative nonangial pain after cardiac surgery: a 2-year prospective multicentre study. CMAJ. 2014;186(7):213-23.

12. Araújo HVS, Figueiredo TR, Costa CRB, Silveira MMBM, Belo RMO, Bezerra SMMS. Quality of life of patients who undergone myocardial revascularization surgery. Rev Bras Enferm [Internet]. 2017;70(2):257-64.

13. Alberte JSP, Ruscalleda RMI, Guariento ME. Qualidade de vida e variáveis associadas ao envelhecimento patológico. Rev Soc Bras Clin Med. 2015 jan-mar;13(1):32-9.

14. Souza AO, Yamaguchi MU. Adesão e não adesão dos idosos ao tratamento antihipertensivo. Saúde Pesq[Internet]. 2015[cited 2016 Jan 29];8(spe):113-22. Available from: http://periodicos.unicesumar.edu.br/index.php/ saudpesq/article/view/3769/2518.

15. Chaves G, Britez N, Munzinger J, Uhlmann L, Gonzalez G, Oviedo G, et al. Education to a healthy lifestyle improves symptoms and cardiovascular risk factors: AsuRiesgo Study. Arq Bras Cardiol[Internet]. 2015[cited 2016 Feb 01];104(5):347-55. Available from: http:// www.scielo.br/pdf/abc/v104n5/0066-782Xabc-20150021.pdf.

16. Sampaio JKVR, Figueiredo Neto JA De, Queiroz LLC, Sousa RML De, Reis LMCB, Silva FMAM. Impacto na Qualidade de Vida Pós-angioplastia Coronariana ou Revascularização do Miocárdio. Rev Bras Cardiol. 2013 Set;26(5):337-46.

17. Lima, KCS, Lima AMF, Torres MV, Xavier CL, Gardenghi G, Moraes I. Qualidade de vida em indivíduos cardiopatas pré e pós-cirurgia cardíaca. Revista Pesquisa em Fisioterapia. 2017 Maio; 7(2): 171-8.

18. Kurfist V, Mokráček A, Krupauerová M, Canádyová J, Bulava A, Pešl L, et al. Health-related quality of life after cardiac surgery - the effects of age, preoperative conditions and postoperative complications. Journal of Cardiothoracic Surgery. 2014; 9(46):1-8. 


\section{Anexos}

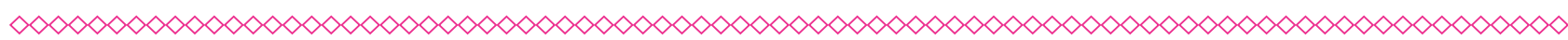

Tabela 1 - Características sociodemográficas da população em estudo ( $\mathrm{n=70)}$, Passo Fundo/RS, 2015.

$\begin{array}{lcc}\text { Variável } & \text { Estatística } \\ \text { Sexo masculino } & & 59,9 \pm 14,0 \\ \text { Estado civil } & & 44(62,9 \%) \\ & & \\ & \text { Solteiro } & 5(7,1 \%) \\ & \text { Casado } & 47(67,1 \%) \\ & \text { Divorciado } & 6(8,6 \%) \\ & \text { Viúvo } & 9(12,9 \%) \\ & \text { Outro } & 3(4,3 \%)\end{array}$

Procedência

$\begin{array}{lc}\text { Passo Fundo } & 7(10,0 \%) \\ \text { Outra-RS } & 60(85,7 \%) \\ \text { SC } & 3(4,3 \%)\end{array}$

Aposentado

$\begin{array}{ll}\text { Sim } & 48(68,6 \%) \\ \text { Não } & 22(31,4 \%)\end{array}$

Valores expressam mediana (p25 - p75). Valor próximo a zero indica pior qualidade de vida. Valor próximo a 100 indica melhor qualidade de vida.

Fonte: Elaborada pelo autor.

(clique para voltar ao texto) 
Tabela 2 - Características clínicas da população em estudo (n=70), Passo Fundo/RS, 2015.

\begin{tabular}{|c|c|}
\hline Variável & $\mathrm{n}^{\circ}(\%)$ \\
\hline $\begin{array}{c}\text { Diabetes } \\
\text { Sim } \\
\text { Não }\end{array}$ & $\begin{array}{l}22(31,4) \\
48(68,6)\end{array}$ \\
\hline $\begin{array}{c}\text { Hipertensão } \\
\text { Sim } \\
\text { Não }\end{array}$ & $\begin{array}{l}47(67,1) \\
32(32,9)\end{array}$ \\
\hline $\begin{array}{l}\text { DPOC } \\
\text { Sim } \\
\text { Não }\end{array}$ & $\begin{array}{c}5(7,1) \\
65(92,9 \%)\end{array}$ \\
\hline $\begin{array}{l}\text { IRC } \\
\text { Sim } \\
\text { Não }\end{array}$ & $\begin{array}{c}7(10,0) \\
63(90,0)\end{array}$ \\
\hline $\begin{array}{l}\text { DAC } \\
\text { Sim } \\
\text { Não }\end{array}$ & $\begin{array}{l}30(42,9) \\
40(57,1)\end{array}$ \\
\hline Tabagista & \\
\hline Não & $44(62,9 \%)$ \\
\hline Sim & $8(11,4 \%)$ \\
\hline Ex-tabagista & $18(25,7 \%)$ \\
\hline
\end{tabular}

DPOC: Doença Pulmonar Obstrutiva Crônica; IRC: Insuficiência Renal Crônica; DAC: Doença Arterial Coronariana. Valores expressam frequência absoluta e relativa.

Fonte: Elaborada pelo autor. 
Tabela 3 - Qualidade de vida pré-cirurgia, pós-cirurgia e no seguimento de três meses ( $n=70)$, Passo Fundo/RS, 2015.

\begin{tabular}{|c|c|c|c|c|}
\hline & \multicolumn{4}{|c|}{ Tempo } \\
\hline & Pré-cirurgia & Pós-cirurgia & $\begin{array}{l}\text { Seguimento } \\
\text { de três meses }\end{array}$ & $\mathbf{P}$ \\
\hline $\begin{array}{l}\text { Capacidade } \\
\text { funcional }\end{array}$ & $40,0(25,0-70,0)$ & $15,0(0-35,0)$ & $70,0(50,0-80,0)$ & $p<0,001$ \\
\hline $\begin{array}{l}\text { Limitação por } \\
\text { aspectos físicos }\end{array}$ & $0(0-25,0)$ & $0(0-25,0)$ & $25,0(0-100,0)$ & $p<0,001$ \\
\hline Dor & $52,0(30,0-62,0)$ & $32,0(22,0-54,2)$ & $62,0(51,0-88,0)$ & $\mathrm{p}<0,001$ \\
\hline $\begin{array}{l}\text { Estado geral de } \\
\text { saúde }\end{array}$ & $80,0(62,0-87,0)$ & $77,0(57,0-87,0)$ & $87,0(67,0-92,0)$ & $p=0,004$ \\
\hline Vitalidade & $50,0(30,0-66.2)$ & $50,0(35,0-66,2)$ & $70,0(50,0-80,0)$ & $p<0,001$ \\
\hline Aspectos sociais & $62,5(34,3-87,5)$ & $50,0(34,3-75,0)$ & $87,5(62,5-100,0)$ & $p<0,001$ \\
\hline $\begin{array}{l}\text { Limitação } \\
\text { por aspectos } \\
\text { emocionais }\end{array}$ & $33,3(0-100,0)$ & $0(0-66,6-66,7)$ & $100,0(67,0-100,0)$ & $p<0,001$ \\
\hline Saúde mental & $64,0(45,0-84,0)$ & $68,0(44,0-80,0)$ & $76,0(67,0-92,0)$ & $\mathrm{p}<0,001$ \\
\hline
\end{tabular}

Valores expressam mediana $\left(p_{25}-p_{75}\right)$. Valor próximo a zero indica pior qualidade de vida. Valor próximo a 100 indica melhor qualidade de vida.

Fonte: Elaborada pelo autor.

(clique para voltar ao texto) 